\title{
Zero-Knowledge User Authentication: An Old Idea Whose Time Has Come
}

\section{Conference Paper}

Author(s):

Chuat, Laurent (1); Plocher, Sarah; Perrig, Adrian

Publication date:

2019-04

Permanent link:

https://doi.org/10.3929/ethz-b-000392315

Rights / license:

In Copyright - Non-Commercial Use Permitted 


\title{
Zero-Knowledge User Authentication: An Old Idea Whose Time Has Come
}

\author{
Laurent Chuat, Sarah Plocher, and Adrian Perrig \\ ETH Zurich
}

\begin{abstract}
User authentication can rely on various factors (e.g., a password, a cryptographic key, biometric data) but should not reveal any secret or private information. This seemingly paradoxical feat can be achieved through zero-knowledge proofs. Unfortunately, naive passwordbased approaches still prevail on the web. Multi-factor authentication schemes address some of the weaknesses of the traditional login process, but generally have deployability issues or degrade usability even further as they assume users do not possess adequate hardware. This assumption no longer holds: smartphones with biometric sensors, cameras, short-range communication capabilities, and unlimited data plans have become ubiquitous. In this paper, we show that, assuming the user has such a device, both security and usability can be drastically improved using an augmented password-authenticated key agreement (PAKE) protocol and message authentication codes.
\end{abstract}

\section{Introduction}

User authentication still typically involves transmitting a plaintext password to the web server, which is problematic for many reasons. First, an attacker could obtain the user's password using malware, a keystroke logger, phishing, or a man-in-the-middle attack. Second, the user might be using the same password on different websites, allowing a malicious server operator to impersonate the user. Third, if the website's database is breached, although passwords may be hashed, a brute-force attack could reveal low-entropy passwords. Fourth, as passwordreuse and low-entropy passwords are problematic, users are constantly advised to pick new, long, complicated passwords. Finally, transmitting the password over a secure channel, storing it as a hash, and using a random salt as well as a strong cryptographic hash function are recommended measures, but they are impossible to enforce or even verify for the end user. The unsalted SHA-1-hashed passwords of millions of LinkedIn users were disclosed in 2012 [8] and hundreds of millions more were disclosed (with corresponding email addresses) in 2016 [5], for example.

Two-factor authentication (2FA) supposedly reinforces standard passwords. Typically on today's web, the time-based one-time password (TOTP) algorithm [10] is used to generate a new 6-digit code every 30 seconds, based on the current time and a secret shared between the server and the user's authentication device (i.e., either a dedicated token or a smartphone). Usually, the user 
must submit their one-time code after the traditional username/password step. For this reason, two-factor authentication is also referred to as "2-step verification" [6].

Although 2FA may protect users against certain attacks, it doesn't completely prevent them. Schneier [12] claims that it "doesn't solve anything". Indeed, an attacker could still obtain the password and a one-time code - or hijack the session - through a real-time attack [13]. The attacker could then "make any fraudulent transaction he wants", which includes changing the password and deactivating 2FA to gain long-term control over the user's online account. Passwords are still weak and typed on untrusted devices. Servers still receive plaintext passwords. Password re-use is still problematic. All secret values may still be known by the server. Finally, assuming that an attacker manages to uncover a large number of passwords, the entropy of a 6-digit code is too low to rule out a brute-force attack. Therefore, TOTP-based authentication alleviates neither website owners nor users from the consequences of password leaks.

Herein we challenge some of the assumptions underlying existing schemes. For example, should we still assume that authentication devices have no Internet access? Clearly, the fact that a smartphone may not have Internet access at all times should be taken into account, but such a situation has become the exception rather than the rule. Also, is it sufficient to supplement a weak form of authentication with a "second step"? We believe, on the contrary, that user authentication should be completely reassessed.

Besides security, usability is actually deteriorated by schemes based on the TOTP algorithm. Every time users want to $\log$ in, they must type an everchanging code displayed on their device - in addition to their password. The scheme we present, ZeroTwo, on the other hand, would fit inside a streamlined process, where the user only enters a username or email address on the website and then approves the login/authorization request in a single step on their smartphone. ZeroTwo relies on an augmented PAKE ${ }^{1}$ protocol and message authentication codes (MACs) to provide the server with a zero-knowledge password proof (ZKPP) and evidence that the legitimate user explicitly authorized critical actions.

Another problem that has been largely overlooked in previous work is that users share their credentials with friends and family [15]. This problem can be solved by moving critical parts of the authentication process to the user's smartphone, which allows them to remotely give someone they trust a restricted, temporary, and revokable access to their online account.

\section{Protocol Overview}

In this section, we provide an overview of how users can authenticate and explicitly authorize actions using ZeroTwo. We chose to base our scheme upon SRP [20] because mature implementations are available. We also borrowed concepts from

\footnotetext{
${ }^{1}$ also referred to as asymmetric password-authenticated key establishment or aPAKE
} 
AugPAKE [14]. In principle, however, any PAKE protocol (e.g., OPAQUE [7]) could be used to develop a scheme similar to ours.

\subsection{Notation}

Table 1 shows the notation we use throughout the paper. The values $n$ and $g$ are agreed upon beforehand. All arithmetic is performed modulo $n . \mathrm{H}()$ and $\mathrm{H}_{K}()$ denote a hash function and an HMAC, respectively.

Table 1. Notation Summary

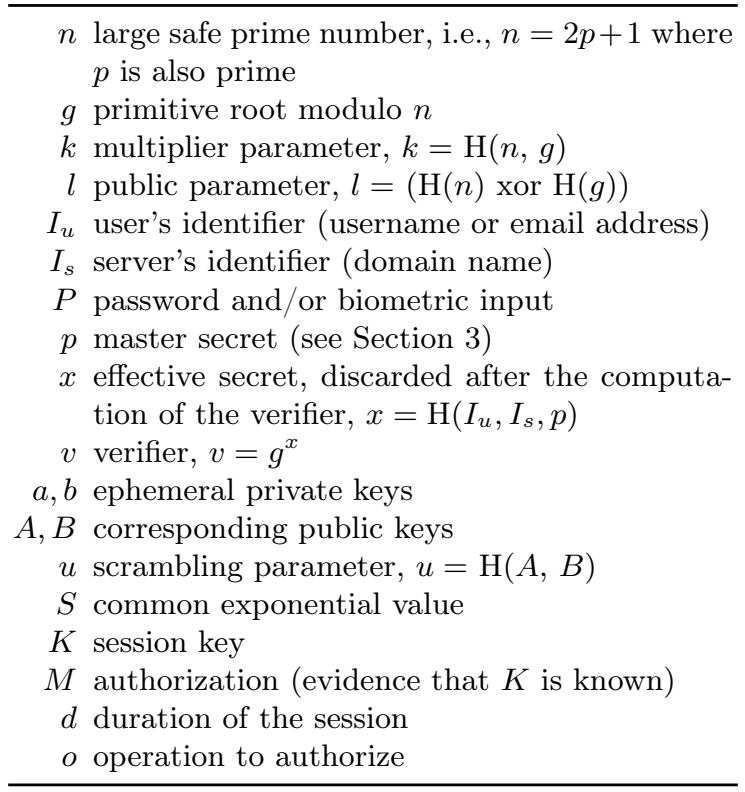

\subsection{Initialization}

During sign up, the following operations must be performed:

1. The user chooses a unique identifier $I_{u}$ and sends it to the server (from a browser).

2. The server replies with a $\mathrm{QR}$ code containing both the user identity $I_{u}$ and the server identity $I_{s}$ as well as the URL that the smartphone must use to send the initialization data.

3. The user scans the QR code; chooses a master secret $p$ (see Section 3), if this has not been done before; and the app computes the verifier $v$ from $x$ :

$$
x=\mathrm{H}\left(I_{u}, I_{s}, p\right)^{2}
$$

${ }^{2}$ As in AugPAKE [14] and other previous work [1], but unlike in SRP [20], using a salt is unnecessary here, as the effective secret is derived from unique identifiers. 


$$
v=g^{x}
$$

4. The user's identity and the verifier are sent to the previously received URL.

5. The server stores the verifier permanently (if the identifier is valid and not already used) under the user's identifier. If the identifier is an email address, the server should first verify that the user has access to that address.

\subsection{Authentication}

The authentication process works as follows:

1. The user initiates a login attempt by sending their identifier $I_{u}$ to the server (from a browser).

2. The server looks up the user's verifier $v$, generates a random ephemeral private key $b$, and computes the corresponding public key:

$$
B=k v+g^{b}
$$

3. The server sends its public key $B$ as well as identifiers $I_{u}$ and $I_{s}$ to both the browser and smartphone. In the common case, the smartphone is connected to the Internet and receives $B$ (with a notification), a fingerprint $[4,17]$ of the public key is displayed on both the smartphone app and the browser, and the user is asked to make sure that the two fingerprints match. If the smartphone is not connected to the Internet, the user must select a method for transferring $B$ from the browser to the app (see Section 2.6).

4. The user can accept the authentication request on their smartphone by entering a password $P$ and/or using an embedded biometric sensor. The master secret $p$ is then used to compute the session key $K$, and $M$, which constitutes evidence that the user authorized a session to be established for a time period $d$ :

$$
\begin{gathered}
x=\mathrm{H}\left(I_{u}, I_{s}, p\right) \\
a=\operatorname{random}() \\
A=g^{a} \\
u=H(A, B) \\
S=\left(B-k g^{x}\right)^{(a+u x)} \\
K=\mathrm{H}(S) \\
M=\mathrm{H}_{K}\left(l, I_{u}, I_{s}, A, B, d\right)
\end{gathered}
$$

5. The user's identifier $I_{u}$, public key $A$, and proof $M$, as well as the authorized duration of the session $d$ are transmitted to the server by the smartphone.

6 . The server verifies $M$ by computing its own session key:

$$
\begin{gathered}
S=\left(A v^{u}\right)^{b} \\
K=\mathrm{H}(S)
\end{gathered}
$$

If the received data is correct, the server automatically redirects the client to the page that required authentication. 


\subsection{Explicit Authorization}

Following the principle of least privilege and to mitigate session hijacking, web developers using ZeroTwo may choose to define a set of actions for which explicit authorization from the user is required. When such an action is requested by the client, the server generates a human-readable message $o$ describing the action and sends it directly to the smartphone, with a notification and a random nonce $c$ (for replay prevention). The smartphone then computes an authorization message $M$ with the session key $K$ :

$$
M=\mathrm{H}_{K}(o, c)
$$

and sends it to the server. This can also be performed through alternative channels, as described in Section 2.6.

This general concept is sometimes referred to as "transaction signing". In the context of this paper, however, because a shared key is established between the client and the server, symmetric cryptography is sufficient.

\subsection{Session Management}

The session key $K$ is stored on the smartphone and considered valid by the server for as long as the session is valid (specified with $d$ ).

If the user wants to terminate the session before it expires automatically, then they can send an authenticated logout message $M$ to the server:

$$
M=\mathrm{H}_{K}(o), \quad \text { where } o=\text { logout. }
$$

The user should also be able to logout in the usual way, i.e., from the web browser, but the above method allows the user (who forgot to logout or was prevented from doing so by an attacker, for example) to terminate the session from their smartphone.

\subsection{Alternative Channels}

A bidirectional communication channel between the smartphone and the server is needed during the authentication and authorization procedures. The server's public key $B$ can simply be displayed as a QR code in the browser and scanned with the ZeroTwo app. Sending data back to the server from the smartphone is more challenging, because it may not be possible to establish a direct Internet connection. The WebRTC standard, through a collection of protocols and APIs, allows modern web browsers to access peripheral hardware, which provides several solutions to our problem:

- Webcam: A camera connected to or embedded in the terminal (e.g., laptop) is used to scan a QR code displayed on the smartphone.

- Bluetooth: A Bluetooth connection is established between the smartphone and the browser to transfer the authentication data.

The user may initiate the authentication process directly from a mobile browser. In that case, the above methods cannot be used. By definition, however, the smartphone would need an Internet connection to start the authentication procedure from the login page and thus an alternative channel would not be needed. 


\section{$3 \quad$ Nature of the Master Secret}

One of the advantages of augmented PAKE protocols is that they provide a great deal of flexibility with regard to the nature of the secret they rely upon. In other words, the master secret (which we denote $p$ ), although it is traditionally assumed to be a password, can be arbitrary long and of any nature. And because of the zero-knowledge property of our protocol, no entity can learn anything about the secret aside from the one who created it. Therefore, the responsibility of developing a process for generating the master secret that is both secure and convenient for users lies entirely with the developers of compatible apps.

Our preferred approach consists in generating a random passphrase (e.g., composed of lowercase, hyphen-separated words). A passphrase has the advantage that it can be memorized and/or written on a piece of paper to be kept in a secure location. Moreover, a long passphrase generated at random from a large set of words has enough entropy to make brute-force and dictionary attacks infeasible. The passphrase can be re-used for several websites because the effective secret $x$ is domain-dependent and the entropy of the master secret is high. The only disadvantage of a passphrase would be to type it at every login, but it can be stored on the smartphone.

One could also combine several secrets. Concatenating a key and a password, for example, would result in a multi-factor scheme. If the same combination is used on multiple websites, however, changing the password is inconvenient as all verifiers must be updated. Therefore, it is not clear whether the presumed security gain would be worth the decrease in usability. Instead, we propose to protect a high-entropy secret with another factor, as described in Section 4.

A single high-entropy secret is sufficient to protect multiple websites. However, to avoid a single point of failure, some users might choose to generate multiple secrets: one for personal websites and one for professional purposes, for example. Therefore, compatible apps should offer an option to manage multiple accounts.

\section{Access Control, Storage, and Backup}

If the master secret is stored on the smartphone, then the access to the ZeroTwocompatible app and the storage of the secret should be protected. The two main options at our disposal are the following:

- Biometric protection: Fingerprint and facial scanners are becoming commonplace on high-end smartphones. Android and iOS both provide APIs for letting developers protect their app with embedded biometric sensors.

- Password protection: Although passwords do not allow for the same ease of use as biometrics, they offer another advantage: the master secret can be encrypted before a backup (in cloud storage, for example).

Although we favor the biometrics approach, a password can be used as a fallback in case the smartphone does not have a biometric sensor, or in case the 
biometric sensor produces a false negative. As for the backup strategy, assuming the master secret is a passphrase, users should be able to decide whether they want to only display the passphrase when it is first generated (to memorize it or write it down) or back it up using a protected cloud storage solution.

We note that a single-secret approach, as opposed to a naive password-based scheme, scales to an arbitrary number of websites without having to constantly remember/backup new secrets, which is particularly important should the user decide to use an offline backup solution.

\section{Comparative Evaluation and Related Work}

The last couple of decades has seen a plethora of proposals for user authentication. In general, existing schemes suffer from at least one of the following drawbacks: (a) they require a dedicated device, (b) they are proprietary, (c) they involve a shared secret, and/or (d) they still require a traditional password. Herein we only discuss a small subset of schemes and refer to the paper by Bonneau et al. [2] for an extensive evaluation of related work. Using their framework we evaluated ZeroTwo and present the results in Table 2.

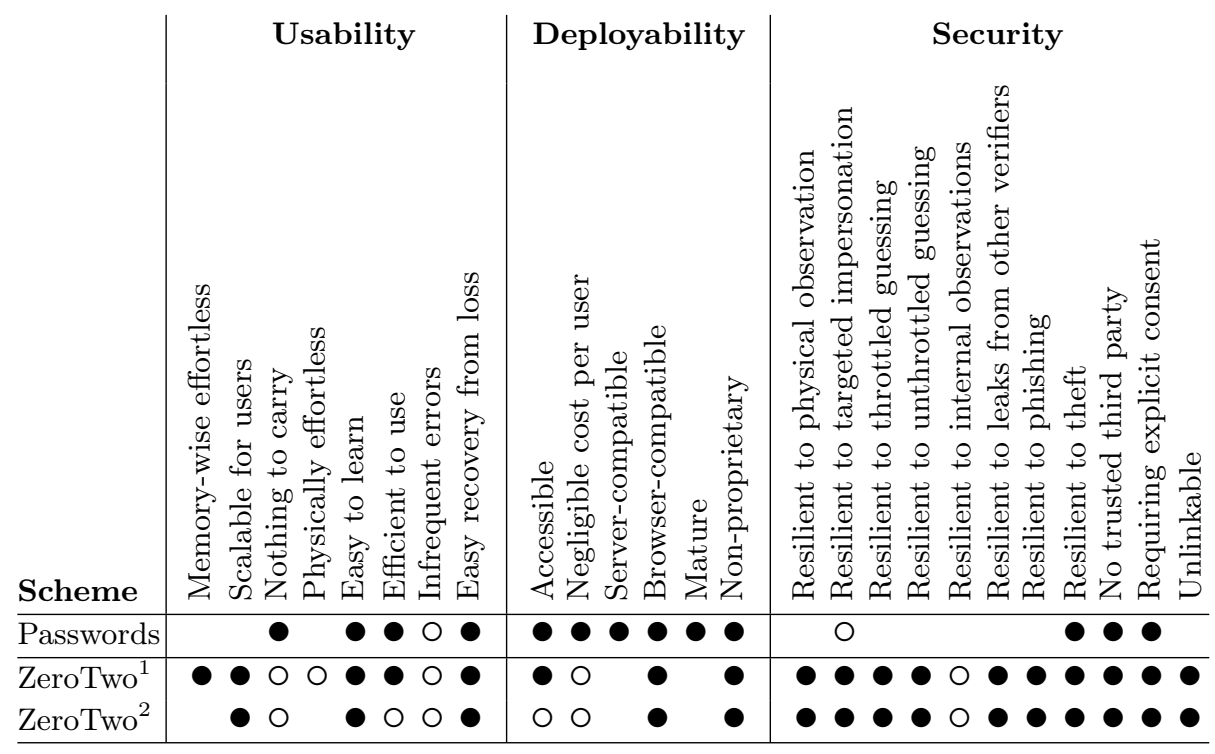

- = offers the benefit; $\circ=$ almost offers the benefit

Table 2. Comparative evaluation of ZeroTwo, assuming a single passphrase is used as the master secret. ${ }^{1}$ Best-case scenario: The smartphone has a biometric sensor to protect the secret and Internet connectivity is available. ${ }^{2}$ Worst-case scenario: The passphrase is protected with a password; the smartphone has no biometric sensor and no Internet connectivity. 
Bonneau [1] had previously proposed a password-based authentication protocol, designed to avoid revealing the password to the server (using Javascript), which requires neither a software update on the client side nor a separate authentication device. Thomas et al. [19] similarly focused on restrictions imposed by legacy systems to address the issues of weak passwords.

Hardware tokens (such as YubiKey [21] or Pico [16]) have the advantage that they are shielded from remote attackers. Although they are particularly adapted to professional contexts, we believe that they are less suited to the general public as many users are unwilling to spend money for a dedicated device.

Cronto [11] is a phone-based scheme close to our proposal in terms of offered features, but it is a proprietary product whose exact design is not publicly known, which makes it hard to analyze and unlikely to be widely deployed outside of the banking industry. SQRL (pronounced "squirrel") or Secure Quick Reliable Login [3] also offers a strong phone-based authentication solution, but it does not support transaction signing or explicit authorization.

Sound-Proof [9] is a recent system that relies on sound for the smartphone and browser to communicate. One of the main goals of Sound-Proof is to provide a seamless experience to users, i.e., the phone need not even be handled for the authentication process to complete. However, the user still has to type a password in the browser, which comes with the issues we discussed previously. Moreover, the complete seamlessness of Sound-Proof is not compatible with our view that certain actions should be explicitly authorized on a trusted device.

The FIDO Alliance has published a number of protocol, framework, and API specifications [18] for user authentication on the web, based on public-key cryptography. To the best our knowledge, none of these specifications rely on password-authenticated key establishment.

\section{Conclusion}

Many forms of user authentication on today's web suffer from severe drawbacks. Using public-key cryptography for authentication is not a novel idea. But whereas asymmetric cryptography is commonly used for server authentication on the web (through TLS), it is not a widespread approach for user authentication. There are several reasons for this: key management is difficult; users need a trusted device with storage, communication, and computation capabilities; and the authentication process must be fast and convenient. We believe, however, that the democratization of smartphones with embedded biometric sensors, unlimited cellular data plans, and new communication standards such as WebRTC now make asymmetric protocols a viable option for user authentication.

\section{Acknowledgments}

We gratefully thank Eduardo Solana for his valuable input in the early stages of this project, Daniel R. Thomas for his extensive feedback, and all the workshop attendees who participated in the discussion and helped improve this paper. 


\section{References}

1. Joseph Bonneau. Getting web authentication right: A best-case protocol for the remaining life of passwords. In Proceedings of the 19th International Workshop on Security Protocols, 2011.

2. Joseph Bonneau, Cormac Herley, Paul C. van Oorschot, and Frank Stajano. The quest to replace passwords: A framework for comparative evaluation of web authentication schemes. In Proceedings of the 33rd IEEE Symposium on Security and Privacy (SEP), 2012.

3. Gibson Research Corporation. SQRL secure quick reliable login. https://www . grc.com/sqrl/sqrl.htm.

4. Sergej Dechand, Dominik Schürmann, Karoline Busse, Yasemin Acar, Sascha Fahl, and Matthew Smith. An empirical study of textual key-fingerprint representations. In Proceedings of the 25th USENIX Security Symposium, 2016.

5. Lorenzo Franceschi-Bicchierai. Another day, another hack: 117 million LinkedIn emails and passwords. https://perma.cc/6MC6-EVHH, May 2016. Motherboard.

6. Google. 2-step verification. https://www.google.com/landing/2step.

7. Stanislaw Jarecki, Hugo Krawczyk, and Jiayu Xu. OPAQUE: An asymmetric PAKE protocol secure against pre-computation attacks. In Proceedings of the 37th Annual International Conference on the Theory and Applications of Cryptographic Techniques (Eurocrypt), 2018.

8. Poul-Henning Kamp. LinkedIn password leak: Salt their hide. ACM Queue, 10(6):20, June 2012.

9. Nikolaos Karapanos, Claudio Marforio, Claudio Soriente, and Srdjan Capkun. Sound-Proof: Usable two-factor authentication based on ambient sound. In Proceedings of the 24th USENIX Security Symposium, 2015.

10. David M'Raihi, Salah Machani, Mingliang Pei, and Johan Rydell. TOTP: Timebased one-time password algorithm. RFC 6238, May 2011.

11. OneSpan. CRONTO mobile app. https://perma.cc/THZ6-3YFW.

12. Bruce Schneier. Two-factor authentication: too little, too late. Communications of the ACM, 48(4):136, April 2005.

13. Bruce Schneier. Real-time attacks against two-factor authentication. https:// perma.cc/FQ9R-USG6, December 2018. Schneier on Security.

14. SeongHan Shin and Kazukuni Kobara. Efficient augmented password-only authentication and key exchange for IKEv2. RFC 6628, June 2012.

15. Supriya Singh, Anuja Cabraal, Catherine Demosthenous, Gunela Astbrink, and Michele Furlong. Password sharing: Implications for security design based on social practice. In Proceedings of the SIGCHI Conference on Human Factors in Computing Systems, 2007.

16. Frank Stajano. Pico: No more passwords! In Proceedings of the 19th International Workshop on Security Protocols, 2011.

17. Joshua Tan, Lujo Bauer, Joseph Bonneau, Lorrie Faith Cranor, Jeremy Thomas, and Blase Ur. Can unicorns help users compare crypto key fingerprints? In Proceedings of the SIGCHI Conference on Human Factors in Computing Systems, 2017.

18. The FIDO Alliance. Specifications overview (FIDO2, WebAuthn, FIDO UAF, FIDO U2F). https://fidoalliance.org/specifications.

19. Daniel R. Thomas and Alastair R. Beresford. Better authentication: Password revolution by evolution. In Proceedings of the 22th International Workshop on Security Protocols, 2014. 
20. Thomas Wu. SRP-6: Improvements and refinements to the Secure Remote Password protocol. IEEE P1363 Working Group, October 2002.

21. Yubico. YubiKey strong two factor authentication for business and individual use. https://www. yubico.com. 